\title{
Two-fluid model for the breakdown of flow alignment in nematic liquid crystals
}

\author{
Helmut R. Brand $\odot^{1,2, *}$ and Harald Pleiner $\odot^{2, \dagger}$ \\ ${ }^{1}$ Department of Physics, University of Bayreuth, 95440 Bayreuth, Germany \\ ${ }^{2}$ Max Planck Institute for Polymer Research, 55021 Mainz, Germany
}

(Received 9 November 2020; accepted 5 January 2021; published 19 January 2021)

\begin{abstract}
We present a macroscopic two-fluid model to explain the breakdown of flow alignment in nematic liquid crystals under shear flow due to smectic clusters. We find that the velocity difference of the two fluids plays a key role to mediate the time-dependent behavior as soon as a large enough amount of smectic order is induced by flow. For the minimal model it is sufficient to keep the nematic degrees of freedom, the mass density of the smectic clusters and the degree of smectic order, the density, and two velocities as macroscopic variables. While frequently a smectic $\mathrm{A}$ or $\mathrm{C}$ phase arises at lower temperatures, this is not required for the applicability of the present model. Indeed, as pointed out before by Gähwiller, there are compounds showing a breakdown of flow alignment over a large temperature range and no smectic phase, but a solid phase at lower temperatures. We also demonstrate that, using a one velocity model, there is no coupling under shear flow between induced smectic order and the director orientation in stationary situations thus rendering such a model to be unsuitable to describe the breakdown of flow alignment. In a two-fluid description, flow alignment breaks down and becomes unstable with regard to a space- and time-dependent state due to an induced finite velocity difference. In an Appendix we outline a mesoscopic model to account for the sign change in the anisotropy of the electric conductivity observed in nematics with smectic clusters.
\end{abstract}

DOI: 10.1103/PhysRevE.103.012705

\section{INTRODUCTION}

Macroscopic two-fluid descriptions have been applied to a variety of complex fluids and soft matter materials composed of two immiscible subsystems. Systems include two immiscible liquids [1,2], for example, concentrated emulsions and colloidal suspensions, two immiscible compound materials in solids and gels [3], and combinations of an ordinary or viscoelastic liquid with a nematic liquid crystal [1]. This approach has also been generalized quite recently to bioinspired materials $[4,5]$.

A general framework using macroscopic dynamics, linear irreversible thermodynamics and symmetry properties has been set up to derive systematically a two-fluid description of complex materials [1]. Most recently this concept has been applied to two-fluid effects in magnetorheological fluids (MRFs) for which many of the macroscopic properties can be tuned continuously in a small to moderate magnetic field [6].

The classical examples for the application of two-fluid descriptions historically are the superfluid phases of ${ }^{4} \mathrm{He}[7,8]$ and ${ }^{3} \mathrm{He}$ [9-13]. In this case there are two truly hydrodynamic

\footnotetext{
*brand@uni-bayreuth.de

†pleiner@mpip-mainz.mpg.de
}

Published by the American Physical Society under the terms of the Creative Commons Attribution 4.0 International license. Further distribution of this work must maintain attribution to the author(s) and the published article's title, journal citation, and DOI. Open access publication funded by the Max Planck Society. velocities due to the broken gauge symmetry, which gives rise to the superfluid velocity $[7,8,14]$. There is, however, one key difference to the two-fluid descriptions of normal fluid complex liquids with two velocities: in this case there is only one truly hydrodynamic velocity, namely, the barycentric velocity. Other combinations of the two velocities such as the velocity difference relax on a long, but finite timescale.

Here we will investigate the macroscopic properties of a nematic liquid crystal with smectic clusters, which can lead to a qualitative change in the macroscopic properties of the nematic. While in ordinary nematics without smectic clusters there is a phenomenon called flow alignment [14-18], this behavior is replaced by a time-dependent behavior of the director in two or three spatial dimensions. By flow alignment one describes the scenario of a planar nematic sample under shear flow for which the director moves away from the planar alignment to include a finite angle with the plates and the normal to the plates. This can arise as a stationary situation and the so-called flow alignment angle is found to be independent of the shear rate experimentally and theoretically for low molecular weight nematics [14-20]. In the presence of smectic clusters flow alignment is replaced by a time-dependent, frequently irregular motion of the director denoted as tumbling or breakdown of flow alignment [21-29]. In addition, in an experiment on the dynamics of point defects it has been shown that smectic clusters can lead to a temporally irregular stick-slip motion of the defect (hedgehog) due to the presence of smectic clusters [30]. Another macroscopic phenomenon observed for a nematic with smectic clusters is a sign change in the anisotropy of the electric conductivity [31-34]. For both experimental observations there is so far no macroscopic or 
mesoscopic model to describe these phenomena. It also turns out that the occurrence of a smectic phase at lower temperatures is not a prerequisite of these phenomena to arise: There are materials that show a breakdown of flow alignment for a temperature range of $50 \mathrm{~K}$ without having a smectic phase at all [22].

In the following we will present a two-fluid model for the breakdown of flow alignment leading to dynamic motion of the director: One fluid is the nematic component and the second fluid corresponds to the smectic clusters. The degree of smectic order enters the picture as a macroscopic variable, which relaxes on a long, but finite timescale. We thus consider two velocities, $v_{i}^{n}$ and $v_{i}^{s}$ for the nematic and the smectic component, respectively, or, equivalently, the barycentric velocity, $v_{i}$ and the velocity difference, $w_{i}=v_{i}^{n}-v_{i}^{s}$. In addition, we have two densities, $\rho_{n}$ and $\rho_{s}$ (or two concentrations $\phi=\rho_{n} / \rho$ and $1-\phi=\rho_{s} / \rho$ with $\left.\rho=\rho_{n}+\rho_{s}\right)$.

As an extra tunable parameter (except for temperature and pressure) of the nematic with smectic clusters one can use an electric field, as it has been pioneered by Skarp's group $[35,36]$, which can be used to shift the boundary between the flow alignment and the tumbling regimes for otherwise constant parameters. Another candidate to tune this boundary continuously are ferromagnetic nematics with smectic clusters in a weak to moderate magnetic field. Flow alignment in ferromagnetic nematics without smectic clusters has been investigated quite recently [37].

The approach presented is actually not restricted to smectic clusters in a nematic, but can be equally applied to a nematic with columnar clusters as they arise for compounds composed of column-forming molecules [38-41]. Another field of application are smectics with bond-orientational order like smectic $I, F$, and $L$ [42-44]. In this case we expect bond-orientational clusters in the associated smectic $C$ phase, which is known theoretically [20] and experimentally [45] to show flow alignment in freely suspended films for the in-plane director.

The paper is organized as follows. In Sec. II, we analyze a model with only one velocity field, the barycentric velocity. The bulk part is dedicated to the study of the macroscopic behavior of systems with two velocity fields. Their description is given in Secs. III A (macroscopic variables), III B (statics), III C (dynamics), III D (dissipative currents), and III E (reversible currents). In Sec. IV we study shear flow in the framework of the two velocity model and demonstrate the breakdown of the stationary, homogeneous flow alignment state. In Sec. IV A we set up the equations under shear flow, and in Secs. IV B and IVC we present a linear stability analysis, first for the smectic density as a conserved quantity and second for the smectic density as a relaxing variable. We summarize by conclusions and perspectives in Sec. V. In Appendix A we present a simple mesoscopic model for the sign change of the anisotropy of the electric conductivity, and in Appendix B we explore the case when the in-plane variations of the smectic density have a wavelength large compared to the sample thickness.

\section{MODEL WITH ONE VELOCITY}

For comparison purposes with the two-fluid model investigated in Sec. III and thereafter, we discuss here a model with one velocity field, $v_{i}$, related to the density of momentum, $g_{i}=\rho v_{i}$, the latter being a conserved quantity. Another relevant variable is the nematic director, $n_{i}$ with $n_{i} \delta n_{i}=0$, related to the spontaneously broken rotational symmetry. Finally, we use the degree of smectic order, $\psi$, associated with the presence of smectic clusters. It is not a truly hydrodynamic variable, but it relaxes on a sufficiently long, but finite timescale. In this section we can assume incompressibility, $\operatorname{div} \boldsymbol{v}=0$, without loss of generality.

In the local formulation of the first law of thermodynamics, the entropy density, $\sigma$, and the energy density, $\varepsilon$, are related to the macroscopic variables by $[46,47]$

$$
T d \sigma=d \varepsilon-v_{i} d g_{i}+h_{i} d n_{i}-\mu_{\psi} d \psi
$$

where the thermodynamic conjugate quantities temperature $T$, the velocity $v_{i}$, the molecular field $h_{i}$ associated with the director, and the conjugate of $\psi, \mu_{\psi}$, are defined via Eq. (1).

For the dynamic equations for the hydrodynamic variables we have

$$
\begin{gathered}
\dot{\sigma}+\nabla_{i} j_{i}^{\sigma D}=2 R / T \\
\dot{g}_{i}+\nabla_{i} p-\lambda_{k j i} \nabla_{j} h_{k}+\beta_{j i} \nabla_{j} \mu_{\psi}+\nabla_{j} \sigma_{i j}^{D}=0, \\
\dot{n}_{i}+\lambda_{i j k} \nabla_{j} v_{k}+Y_{i}^{D}=0, \\
\dot{\psi}+\beta_{i j} \nabla_{i} v_{j}+X^{D}=0
\end{gathered}
$$

with the dots representing time derivatives $\partial / \partial t$, thereby suppressing all transport derivatives, which are irrelevant for the purposes of this section.

Reversible contributions are due to the pressure $p$, due to the well-known nematic flow alignment tensor $\lambda_{i j k}$, and due to a tensor $\beta_{i j}$, introduced by Liu in his macroscopic dynamic description of the nematic-smectic A transition [48]

$$
\begin{gathered}
\lambda_{i j k}=\frac{1}{2}(1-\lambda) \delta_{i j}^{\perp} n_{k}-\frac{1}{2}(1+\lambda) \delta_{i k}^{\perp} n_{j}, \\
\beta_{i j}=\beta_{\|} n_{i} n_{j}+\beta_{\perp} \delta_{i j}^{\perp},
\end{gathered}
$$

with the transverse Kronecker delta $\delta_{i j}^{\perp}=\delta_{i j}-n_{i} n_{j}$.

The dissipation function, $R$, the source term of the dynamic equation of the entropy density, is associated with the second law of thermodynamics, $R>0$,

$$
R=-j_{i}^{\sigma D} \nabla_{i} T-\sigma_{i j}^{D} A_{i j}-Y_{i}^{D} h_{i}+X^{D} \mu_{\psi},
$$

and contains the dissipative currents and quasicurrents

$$
\begin{gathered}
Y_{i}^{D}=-\frac{1}{\gamma} h_{i}, \\
X^{D}=\frac{1}{\tau_{\psi}} \mu_{\psi}, \\
j_{i}^{\sigma D}=-\kappa_{i j} \nabla_{j} T, \\
\sigma_{i j}^{D}=-v_{i j k l} A_{k l},
\end{gathered}
$$

with $A_{i j}=\frac{1}{2}\left(\nabla_{i} v_{j}+\nabla_{j} v_{i}\right)$. 
We further need here the static relation of $\mu_{\psi}$ with $\psi$,

$$
\mu_{\psi}=\chi_{\psi} \delta \psi
$$

Adding the standard textbook expression for the molecular field of nematics, $h_{i}$ [15], the setup of the relevant equations is completed.

Now we proceed quite in parallel to the usual discussion of flow alignment in uniaxial nematics, while additionally taking into account the dynamic equation for the macroscopic variable $\psi$. We look for a stationary flow situation under a simple shear flow $S$ in the $y-z$ plane:

$$
S=\nabla_{y} v_{z} .
$$

We assume that a stationary solution for the director can be found in the $y-z$ plane. Therefore, we make the ansatz for $n_{i}$

$$
n_{i}=\left(\sin \theta_{f}, \cos \theta_{f}\right),
$$

with a constant flow alignment angle $\theta_{f}$.

For the director equation we have for a stationary situation the condition

$$
\varepsilon_{i j k} n_{j} \dot{n}_{k}=-\lambda_{i j k} \nabla_{j} v_{k}+\frac{1}{\gamma} h_{i}=0,
$$

leading to

$$
\cos \left(2 \theta_{f}\right)=1 / \lambda,
$$

the classical result from textbooks [15]. We thus find that in this one velocity model the degree of smectic order is not influencing the flow alignment angle.

Next we investigate the smectic order induced by shear flow. For a stationary situation we obtain from Eq. (5)

$$
\delta \psi_{f}=\frac{\tau_{\psi}}{2 \chi_{\psi}}\left(\beta_{\perp}-\beta_{\|}\right) S \sin \left(2 \theta_{f}\right)
$$

From Eqs. (17) and (18) we infer that in the present onefluid model there is no connection between the flow alignment in a uniaxial nematic and the build-up of smectic order by shear flow; the flow alignment angle given by the flow alignment parameter $\lambda$ is independent of the applied shear rate, $S$, while the induced amount of smectic order is proportional to the shear rate, $S$. We note that taking into account additionally the smectic concentration variable $\phi$ would not lead to any change regarding flow alignment, since $\phi$ does not couple to shear flow, nor to director rotations.

We therefore conclude that to describe the breakdown of stationary flow alignment by smectic clusters, the present onefluid model is insufficient and has to be replaced by a more general framework. To cope with the nonstationary situation $\left(\dot{n}_{i} \neq 0\right)$, we will introduce a two velocity model.

\section{TWO-FLUID MODEL FOR NEMATICS WITH SMECTIC CLUSTERS}

\section{A. Variables}

The hydrodynamics of a nematic liquid crystal is described by the momentum density $g_{i}$, the mass density $\rho$, and the total energy density $\varepsilon$ representing the local conservation laws of a fluid, as well as by the director field $n_{i}$. The latter is associated with the spontaneously broken continuous rotational symmetry.
In the following we will investigate a new two-fluid model for a nematic liquid crystal with smectic clusters.

On the macroscopic level we describe the system as a homogeneous mixture of smectic and nematic parts in the spirit of a binary mixture of different mass particles. The director orientation is assumed to be the same in the nematic and smectic parts. In Sec. IV B of the manuscript the exchange between smectic and nematic parts is described by a diffusive mass transport according to two different velocities. Therefore, the two types of densities are individually conserved quantities. In $\mathrm{Sec}$. IV C we allow for changes by mutual relaxation with the result that only the total momentum is conserved. The degree of smectic order, which is nonzero only in the smectic parts, is on the macroscopic level an averaged relaxational degree of freedom.

We note that in a macroscopic description the size and shape of the smectic clusters enters through the values of the coefficients in the macroscopic dynamic equations, in particular in the equations for the concentration, $\phi$, the relative velocity, $w_{i}$, and the degree of smectic order, $\psi$. Interfacial effects are taken into account along the same lines in an efficient way.

The smectic and the nematic mass density, $\rho_{s}$ and $\rho_{n}$, respectively, add up to the total density $\rho=\rho_{n}+\rho_{s}$. Similarly, the two velocities give rise to a nematic momentum density, $g_{i}^{n}=\rho_{n} v_{i}^{n}$, and to a smectic one, $g_{i}^{s}=\rho_{s} v_{i}^{s}$ that add up to the total momentum density $g_{i}=\rho_{s} v_{i}^{s}+\rho_{n} v_{i}^{n}$, thereby defining the mean velocity $v_{i}=\phi v_{i}^{s}+(1-\phi) v_{i}^{n}=g_{i} / \rho$. For details of these two-fluid aspects, cf. Ref. [1].

As additional variables compared to the one-fluid description, we therefore take the relative velocity, $w_{i}=v_{i}^{s}-v_{i}^{n}$, between the nematic and the smectic velocities, as well as the mass concentration of the smectic clusters, $\phi=\rho_{s} / \rho$. This variable is conserved for incompressible fluids with constant $\rho$ and $\operatorname{div} \boldsymbol{v}=0$.

The first law of thermodynamics relates changes of the variables to changes of the energy density $\varepsilon$ by the Gibbs relation $[46,47]$

$$
\begin{aligned}
d \varepsilon= & T d \sigma+\mu d \rho+\Pi d \phi+\boldsymbol{v} \cdot d \boldsymbol{g} \\
& +\boldsymbol{m} \cdot d \boldsymbol{w}+h_{i} d n_{i}+\mu_{\psi} d \psi .
\end{aligned}
$$

The entropy density $\sigma$ represents the thermal degree of freedom of the system. The appropriate thermodynamic conjugates are the temperature $T$, the chemical potential $\mu$, the osmotic pressure $\Pi$, the mean velocity $v_{i}=g_{i} / \rho$, the molecular field of the director $n_{i}, h_{i}$, and the conjugate fields $m_{i}$ and $\mu_{\psi}$.

From the requirement that the energy of the system is a first order Eulerian form of all extensive variables, one gets for the pressure $p \equiv-(\partial / \partial V) \int \varepsilon d V=-(\partial / \partial V) E$ the GibbsDuhem relation

$$
\begin{aligned}
d p= & \sigma d T+\rho d \mu-\Pi d \phi+\boldsymbol{g} \cdot d \boldsymbol{v} \\
& -\boldsymbol{m} \cdot d \boldsymbol{w}-h_{i} d n_{i}-\mu_{\psi} d \psi .
\end{aligned}
$$




\section{B. Statics}

The static behavior of our macroscopic system is conveniently described by the energy functional

$$
\begin{aligned}
\varepsilon= & \varepsilon_{0}+\frac{T}{2 C_{V}}(\delta \sigma)^{2}+\frac{1}{2 \kappa_{\phi}}(\delta \phi)^{2}+\frac{1}{2 \rho^{2} \kappa_{\mu}}(\delta \rho)^{2} \\
& +\frac{1}{\alpha_{\phi}}(\delta \phi)(\delta \sigma)+\frac{1}{\rho \alpha_{\rho}}(\delta \rho)(\delta \sigma)+\frac{1}{\rho \kappa_{\pi}}(\delta \rho)(\delta \phi) \\
& +\frac{1}{2} K_{i j k l}\left(\nabla_{j} n_{i}\right)\left(\nabla_{l} n_{k}\right)+\frac{\chi_{\psi}}{2}(\delta \psi)^{2}+\chi_{\rho}(\delta \psi)(\delta \rho) \\
& +\chi_{\sigma}(\delta \psi)(\delta \sigma)+\chi_{\phi}(\delta \psi)(\delta \phi),
\end{aligned}
$$

where $\delta \sigma$ and $\delta \rho$ are deviations from their equilibrium values $\sigma_{0}$ and $\rho_{0}$ and where $\psi$ and $\phi$ are assumed to be zero in equilibrium, although finite values of $\psi$ and $\phi$ due to thermodynamic pretransitional effects would not change the analysis. From Eq. (21) the conjugate fields follow by taking variational derivatives according to the Gibbs relation, Eq. (19),

$$
\begin{gathered}
\delta T=\frac{T}{C_{V}} \delta \sigma+\frac{1}{\alpha_{\phi}} \delta \phi+\frac{1}{\rho \alpha_{\rho}} \delta \rho+\chi_{\sigma} \delta \psi, \\
\mu_{\psi}=\chi_{\psi} \delta \psi+\chi_{\rho} \delta \rho+\chi_{\sigma} \delta \sigma+\chi_{\phi} \delta \phi, \\
\Pi=\frac{1}{\kappa_{\phi}} \delta \phi+\frac{1}{\rho \kappa_{\pi}} \delta \rho+\frac{1}{\alpha_{\phi}} \delta \sigma+\chi_{\phi} \delta \psi \\
+\boldsymbol{w} \cdot \boldsymbol{g}+\rho \boldsymbol{w}^{2}(1-2 \phi), \\
\mu=\frac{1}{\rho^{2} \kappa_{\mu}} \delta \rho+\frac{1}{\rho \kappa_{\pi}} \delta \phi+\frac{1}{\rho \alpha_{\rho}} \delta \sigma+\chi_{\rho} \delta \psi \\
+\boldsymbol{w}^{2} \phi(1-\phi), \\
h_{i}=\frac{\delta E}{\delta n_{i}}=h_{i}^{\prime}-\nabla_{j} \Phi_{i j},
\end{gathered}
$$

with $h_{i}^{\prime}=\partial \varepsilon / \partial n_{i}$ and $\Phi_{i j}=\partial \varepsilon / \partial\left(\nabla_{j} n_{i}\right)$.

There is a total of six static susceptibilities from the binary mixture fluid $\left(C_{V}, \alpha_{\phi}, \alpha_{\rho}, \kappa_{\phi}, \kappa_{\pi}, \kappa_{\mu}\right)$, four are related to the smectic order $\left(\chi_{\psi}, \chi_{\rho}, \chi_{\sigma}\right.$, and $\left.\chi_{\phi}\right)$ and three are the standard nematic coefficients $\left(K_{1,2,3}\right)$.

Finally, the remaining relations between conjugates and variables,

$$
v_{i}=\frac{g_{i}}{\rho} \quad \text { and } \quad m_{i}=\phi(1-\phi) \rho w_{i} \equiv \alpha w_{i},
$$

are not really static, but nevertheless follow from the energy density, in particular from the kinetic energy density $\varepsilon_{\text {kin }}=\left(1 / 2 \rho_{n}\right)\left[\boldsymbol{g}^{(n)}\right]^{2}+\left(1 / 2 \rho_{s}\right)\left[\boldsymbol{g}^{(s)}\right]^{2}=(1 / 2) \boldsymbol{g}^{2}+(\alpha / 2) \boldsymbol{w}^{2}$. The $w_{i}$-dependence of the chemical potential and the osmotic pressure are due to the $\rho$ - and $\phi$-dependence of $\alpha$.

\section{Dynamics}

The dynamical equations for the director and fluid degrees of freedom and for the smectic modulus are

$$
\begin{gathered}
\dot{\epsilon}+\nabla_{i}(\epsilon+p) v_{i}+\nabla_{i}\left(j_{i}^{(\epsilon) R}+j_{i}^{(\epsilon) D}\right)=0, \\
\dot{\sigma}+\nabla_{i}\left(\sigma v_{i}+j_{i}^{(\sigma) R}+j_{i}^{(\sigma) D}\right)=2 R / T, \\
\dot{\rho}+\nabla_{i}\left(\rho v_{i}\right)=0, \\
\dot{\phi}+v_{j} \nabla_{j} \phi+\nabla_{i}\left(\phi(1-\phi) w_{i}+j_{i}^{(\phi) R}+j_{i}^{(\phi) D}\right)=0,
\end{gathered}
$$

$$
\begin{gathered}
\dot{g}_{i}+\nabla_{j}\left(g_{i} v_{j}\right)+\nabla_{j}\left(\sigma_{i j}^{(t h)}+\sigma_{i j}^{R}+\sigma_{i j}^{D}\right)=0 \\
\dot{w}_{i}+v_{j} \nabla_{j} w_{i}+\nabla_{i}\left(\rho^{-1} \Pi\right)+X_{i}^{R}+X_{i}^{D}=0 \\
\dot{\psi}+v_{i} \nabla_{i} \psi+Z^{R}+Z^{D}=0 \\
\dot{n}_{i}+v_{j} \nabla_{j} n_{i}+Y_{i}^{D}+Y_{i}^{R}=0
\end{gathered}
$$

with $2 A_{i j}=\nabla_{i} v_{j}+\nabla_{j} v_{i}$, and $R$ the energy dissipation function. The stress tensor $\sigma_{i j}^{(t h)}=\delta_{i j} p+\Phi_{k j} \nabla_{i} n_{k}$ contains the isotropic pressure and the nematic Ericksen stress. These equations follow from Refs. [1,2,6] and contain, apart from the reversible (superscript $R$ ) and irreversible, dissipative (superscript $D$ ) phenomenological currents, also transport and convection whenever appropriate. The latter are reversible and, indeed, all transport contributions (including the isotropic pressure) add up to zero entropy production. The energy conservation law is redundant due to the Gibbs relation, Eq. (19).

In the whole set of dynamic equations the mean velocity $v_{i}$ has been chosen as the convective velocity for all variables. This ensures zero entropy production of the convective derivatives. Due to various material dependent contributions in the reversible currents (see below), the actual convective velocities can be different from $v_{i}$ and can be specific for the different variables.

For the phenomenological parts of the currents the second law of thermodynamics requires

$$
\begin{aligned}
R= & -j_{i}^{(\sigma) *} \nabla_{i} T-j_{i}^{(\phi) *} \nabla_{i} \Pi-\sigma_{i j}^{*} \nabla_{j} v_{i} \\
& +m_{i} X_{i}^{*}+h_{i} \delta_{i k}^{\perp} Y_{k}^{*}+\mu_{\psi} Z^{*} \geqslant 0,
\end{aligned}
$$

with the equal sign ( $>$ sign) for $*=R(*=D)$.

\section{Dissipative currents}

The dissipative parts of the currents introduced above can be deduced from a potential, the dissipation function $R$, that reads in bilinear approximation

$$
\begin{aligned}
2 R= & \kappa_{i j}\left(\nabla_{i} T\right)\left(\nabla_{j} T\right)+D_{i j}\left(\nabla_{i} \Pi\right)\left(\nabla_{j} \Pi\right) \\
& +D_{i j}^{\psi \psi}\left(\nabla_{i} \mu_{\psi}\right)\left(\nabla_{j} \mu_{\psi}\right)+2 D_{i j}^{\psi \phi}\left(\nabla_{i} \Pi\right)\left(\nabla_{j} \mu_{\psi}\right) \\
& +2 D_{i j}^{T \phi}\left(\nabla_{i} T\right)\left(\nabla_{j} \Pi\right)+2 \kappa_{i j}^{\psi T}\left(\nabla_{i} T\right)\left(\nabla_{j} \mu_{\psi}\right) \\
& +\frac{1}{\tau_{\psi}} \mu_{\psi}^{2}+\xi \boldsymbol{m}^{2}+\frac{1}{\gamma_{1}} h_{i} h_{j} \delta_{i j}^{\perp} \\
& +2 v_{i j k l}^{(c)}\left(\nabla_{j} v_{i}\right)\left(\nabla_{l} m_{k}\right)+v_{i j k l}^{(w)}\left(\nabla_{j} m_{i}\right)\left(\nabla_{l} m_{k}\right) \\
& +v_{i j k l}\left(\nabla_{j} v_{i}\right)\left(\nabla_{l} v_{k}\right),
\end{aligned}
$$

where all second rank tensors are of the uniaxial form $\kappa_{i j}=$ $\kappa_{\|} n_{i} n_{j}+\kappa_{\perp} \delta_{i j}^{\perp}$ and where also the 4-rank material tensors reflect the uniaxial structure and contain five parameters each $[47,49]$.

From Eq. (37) the following dissipative currents are obtained:

$$
\begin{aligned}
j_{i}^{(\sigma) D}= & -(\partial R) /\left(\partial \nabla_{i} T\right) \\
= & -\kappa_{i j} \nabla_{j} T-\phi(1-\phi) d_{i j}^{(T)} \nabla_{j} \Pi \\
& -\kappa_{i j}^{\psi T} \nabla_{j} \mu_{\psi},
\end{aligned}
$$




$$
\begin{gathered}
\sigma_{i j}^{D}=-(\partial R) /\left(\partial \nabla_{j} v_{i}\right) \\
=-v_{i j k l} \nabla_{l} v_{k}-v_{i j k l}^{(c)} \nabla_{l} m_{k}, \\
X_{i}^{D}=(\delta R) /\left(\delta m_{i}\right) \\
=\xi m_{i}-\nabla_{j}\left(v_{i j k l}^{(w)} \nabla_{l} m_{k}+v_{k l i j}^{(c)} \nabla_{l} v_{k}\right), \\
Y_{i}^{D}=(\partial R) /\left(\partial h_{i}\right) \\
j_{i}^{(\phi) D}=\frac{1}{\gamma_{1}} \delta_{i j}^{\perp} h_{j}, \\
=-D_{i j} \nabla_{j} \Pi-\phi(1-\phi) d_{i j}^{(T)} \nabla_{j} T \\
-D_{i j}^{\psi \phi} \nabla_{j} \mu_{\psi}, \\
Z^{D}=(\delta R) /\left(\delta \mu_{\psi}\right) \\
=\frac{1}{\tau_{\psi}} \mu_{\psi}-\nabla_{i}\left(D_{i j}^{\psi \psi} \nabla_{j} \mu_{\psi}\right) \\
-\nabla_{i}\left(D_{i j}^{\psi \phi} \nabla_{j} \Pi+\kappa_{i j}^{\psi T} \nabla_{j} T\right),
\end{gathered}
$$

where thermodiffusion is written in the usual way with $D_{i j}^{(T)}=$ $\phi(1-\phi) d_{i j}^{(T)}$. The relative velocity, $w_{i}$, always relaxes, since it is not related to any broken symmetry, nor to a conservation law. The same reasoning applies to the variable $\psi$. All other variables are conserved or related to broken symmetries and show diffusional behavior.

\section{E. Reversible currents}

For the reversible parts of the currents in Eqs. (31)-(35) we find

$$
\begin{gathered}
j_{i}^{(\sigma) R}=\bar{\beta}_{i j} m_{j} \\
\sigma_{i j}^{R}=2 \beta_{2} m_{i} w_{j}+\beta_{i j} \mu_{\psi}-\lambda_{k j i} h_{k} \\
X_{i}^{R}=\bar{\beta}_{i j} \nabla_{j} T+\gamma_{i j} \nabla_{j} \Pi+\beta_{2} w_{j}\left(\nabla_{i} v_{j}+\nabla_{j} v_{i}\right) \\
+\beta_{3} m_{j}\left(\nabla_{j} w_{i}-\nabla_{i} w_{j}\right)+\beta_{4} w_{j}\left(\nabla_{j} v_{i}-\nabla_{i} v_{j}\right) \\
+\nabla_{j}\left(\hat{\beta}_{j i} \mu_{\psi}\right)-\beta_{1} h_{j} \nabla_{i} n_{j}-\nabla_{j}\left(\lambda_{k j i}^{(m)} h_{k}\right) \\
+\beta_{5} \mu_{\psi} \nabla_{i} \psi, \\
Y_{i}^{R}=-\lambda_{i j k} \nabla_{j} v_{k}-\lambda_{i j k}^{(m)} \nabla_{j} m_{k}+\beta_{1} m_{j} \nabla_{j} n_{i} \\
Z^{R}=\beta_{i j} A_{i j}+\hat{\beta}_{i j} \nabla_{i} m_{j}-\beta_{5} m_{i} \nabla_{i} \psi \\
j_{i}^{(\phi) R}=\gamma_{i j} m_{j}
\end{gathered}
$$

with $\lambda_{i j k}^{(m)}=\frac{1}{2} \lambda_{1}^{(m)} \delta_{i j}^{\perp} n_{k}+\frac{1}{2} \lambda_{2}^{(m)} \delta_{i k}^{\perp} n_{j}, \lambda_{i j k}$ given by Eq. (6) and the rank-2 material tensors by Eq. (7).

These reversible currents add to the reversible $\boldsymbol{v}$-dependent contributions already made apparent in Sec. III C. They can be used to tune the transport and convective velocities of the different variables, cf. Ref. [1], Sec. 6. In particular, the orientation $\dot{n}_{i}$ is transported and convected by the mean velocity, since the $n_{i}$ is the same in both phases. As a result $\beta_{1}=0$ and $\lambda_{1}^{(m)}=\lambda_{2}^{(m)}$. For $\dot{\rho}_{n}$ and $\dot{g}_{i}^{(n)}$ the transport velocity is $v_{i}^{(n)}$, while for $\dot{\rho}_{s}$ and $\dot{g}_{i}^{(s)}$ it is $v_{i}^{(s)}$, resulting in $\gamma_{\perp}=0=\gamma_{\|}$ and $\beta_{2}=\beta_{4}=1 / 2$ and $\beta_{3}=1 / \rho_{s}-1 / \rho_{n}$. For the smectic order $\psi$ the transport velocity is $v_{i}^{(s)}$ leading to $\beta_{5}=-1 / \rho_{s}$. Nonlinear contributions are included where they contribute to the transport and convection of variables.

\section{SHEAR FLOW IN A TWO-FLUID DESCRIPTION}

\section{A. The coupling of all hydrodynamic degrees of freedom under shear mediated by the relative velocity field}

In this section we study the question of flow alignment under external shear flow using the two-fluid model presented in detail in Sec. III. As in Sec. II we will focus on a twodimensional situation with the shear flow applied in the $y-z$ plane.

This assumption of an in-plane director field greatly facilitates the analysis. Clearly a three-dimensional analysis will be useful in the general case leading to out-of-plane director motions. These will play an especially important role in the boundary layers close to the bounding surfaces of the sample.

Nevertheless, already the two-dimensional analysis reveals the basic mechanism for the breakdown of the flow alignment state, but may not allow for quantitative comparisons with experiments.

Therefore, we take for the director field the same ansatz,

$$
n_{i}=(\sin \theta, \cos \theta)
$$

which reads in terms of deviations from the flow alignment state

$$
\delta n_{i}=\left(\cos \theta_{f},-\sin \theta_{f}\right) \delta \theta .
$$

For the imposed shear flow we assume a linear profile,

$$
S=\nabla_{y} v_{z}
$$

where $v_{z}$ is now the barycentric/mean velocity, because this is the quantity which is controlled in a classical shear flow experiment. Deviations of $S$ will be mostly neglected, and in addition we set $v_{x}=0$.

The assumption of a linear velocity profile of the shear velocity in Eq. (52) will become problematic well above the onset of an instability of flow alignment. Therefore, we focus on the linear instability. Well above threshold, that is in the strongly nonlinear regime, deformations of the linear velocity profile can be expected to arise in general, making the whole analysis rather complicated and requiring a full numerical treatment.

In addition we assume that $w_{x}=0$, in agreement with a strictly two-dimensional situation.

Discarding higher order gradient terms associated with the diffusion of $n_{i}$, we then have the dynamic equation for the director field,

$$
\boldsymbol{n} \times\left(\frac{\partial}{\partial t} \boldsymbol{n}+\boldsymbol{Y}^{R}\right)=0
$$

or, explicitly,

$$
\begin{aligned}
& n_{y} \dot{n}_{z}-n_{z} \dot{n}_{y} \\
& \quad=\lambda\left(n_{z}^{2}-n_{y}^{2}\right)\left[\frac{S}{2}+\frac{\lambda^{(m)} \alpha}{2 \lambda}\left(\nabla_{y} w_{z}+\nabla_{z} w_{y}\right)\right]-\frac{S}{2},
\end{aligned}
$$


with $\alpha$ given in Eq. (27). Clearly, this equation reduces to the special case of the one-fluid model by taking $w_{i} \equiv 0$.

In terms of deviations from the flow alignment angle we get

$$
\dot{\theta}=-\lambda S \sin \left(2 \theta_{f}\right) \delta \theta-\frac{\alpha}{2} \lambda^{(m)} \cos \left(2 \theta_{f}\right)\left(\nabla_{y} w_{z}+\nabla_{z} w_{y}\right) .
$$

Next we come to the dynamic equation for the degree of smectic order, $\psi$. For the dynamic equation for $\psi$ we have in lowest order and neglecting the convective term

$$
\dot{\psi}+\frac{\chi_{\psi}}{\tau_{\psi}} \delta \psi+\beta_{i j} A_{i j}+\hat{\beta}_{i j} \alpha \nabla_{j} w_{i}=0,
$$

where the tensor $\beta_{i j}$ has already been discussed in Sec. II and where $\hat{\beta}_{i j}$ represents the two-fluid contribution. Note that $\delta \psi=\delta \psi_{f}+\delta \bar{\psi}$ contains the stationary smectic order induced by the shear flow, $\delta \psi_{f}$, Eq. (18), and the deviations from the flow alignment state, $\delta \bar{\psi}$.

In terms of deviations from the flow alignment state we get

$$
\begin{aligned}
\dot{\psi}+ & \frac{\chi_{\psi}}{\tau_{\psi}} \delta \bar{\psi}-\left(\beta_{\|}-\beta_{\perp}\right) S \cos \left(2 \theta_{f}\right) \delta \theta \\
& +\alpha \hat{\beta}_{\perp}\left(\nabla_{y} w_{y}+\nabla_{z} w_{z}\right) \\
& +\alpha\left(\hat{\beta}_{\|}-\hat{\beta}_{\perp}\right)\left[\left(\cos \theta_{f}\right)^{2} \nabla_{z} w_{z}+\left(\sin \theta_{f}\right)^{2} \nabla_{y} w_{y}\right. \\
& \left.+\frac{1}{2} \sin \left(2 \theta_{f}\right)\left(\nabla_{y} w_{z}+\nabla_{z} w_{y}\right)\right]=0
\end{aligned}
$$

Inspecting Eqs. (54)-(57) we see that, due to $w_{i}$, there is a coupling between the nematic orientation and the smectic order, which is absent in the one-fluid description.

In the dynamic equation for the smectic mass concentration, Eq. (31), we neglect transport terms of the form $v_{i} \nabla_{i} \phi$ and $w_{i} \nabla_{i} \phi$, as well as the cross-diffusive terms in Eq. (42). Taking into account that $\gamma_{i j}=0$ in Eq. (49), we are left with a rather simple equation,

$$
\dot{\phi}+\frac{\alpha}{\rho} \nabla_{i} w_{i}-\frac{1}{\kappa_{\phi}} d_{i j} \nabla_{i} \nabla_{j} \phi=0,
$$

describing the coupling to the divergence of the relative velocity and the smectic mass density diffusion. This coupling provides the indirect coupling of $\phi$ to shear flow and director rotations in the two-fluid description, which is absent in the one-fluid model. In Sec. IV B we will approximate the diffusional term when discussing the oscillating instability, to get an analytical result. Note, in Sec. IVC we will replace this diffusion by a relaxation and show what will be changed.

For the dynamic equation for the velocity difference, $w_{i}$, we have, discarding the convective nonlinearity,

$$
\dot{w}_{i}+\nabla_{i}(\Pi / \rho)+X_{i}^{R}+X_{i}^{D}=0 .
$$

Inserting the lowest order terms from statics, Eq. (24), dissipative dynamics, Eq. (40), and reversible dynamics, Eq. (46), and neglecting the convective term we obtain two equations for $w_{y}$ and $w_{z}$,

$$
\begin{array}{r}
\dot{w}_{y}+\frac{1}{\kappa_{\phi} \rho} \nabla_{y} \phi+\alpha \xi w_{y}+\chi_{\psi} \hat{\nabla}_{y} \delta \bar{\psi}=0, \\
\dot{w}_{z}+\frac{1}{\kappa_{\phi} \rho} \nabla_{z} \phi+\alpha \xi w_{z}+S w_{y}+\chi_{\psi} \hat{\nabla}_{z} \delta \bar{\psi}=0,
\end{array}
$$

with the operators

$$
\begin{aligned}
& \hat{\nabla}_{y}=\left[\hat{\beta}_{\perp}+\hat{\beta}_{a} \sin ^{2}\left(\theta_{f}\right)\right] \nabla_{y}+\frac{1}{2} \hat{\beta}_{a} \sin \left(2 \theta_{f}\right) \nabla_{z}, \\
& \hat{\nabla}_{z}=\left[\hat{\beta}_{\perp}+\hat{\beta}_{a} \cos ^{2}\left(\theta_{f}\right)\right] \nabla_{z}+\frac{1}{2} \hat{\beta}_{a} \sin \left(2 \theta_{f}\right) \nabla_{y} .
\end{aligned}
$$

From Eqs. (61) we see that the externally applied shear flow leads to a coupling of $w_{y}$ and $w_{z}$ via the contribution $\sim S$, while the $\hat{\beta}_{i j}$ tensor provides the coupling to gradients of $\psi$.

Finally, there is the generalized Navier-Stokes Eq. (32) describing the dynamics of the momentum density $g_{i}$. In the flow alignment state the stress tensor is constant and $\dot{g}_{i}=0$. In the general state there is a reversible contribution $\beta_{i j} \chi_{\psi} \delta \bar{\psi}$ to the stress tensor, Eq. (45), which is not constant. In principle this can be compensated by a deviation of the mean velocity $\delta v_{i}$ from its flow alignment state, Eq. (52), via the viscosity contribution. This contribution is irrelevant otherwise.

Thus, we observe that Eqs. (55), (57), (58), (60), and (61) provide us with a closed set of five equations for the five variables $\delta \theta, \delta \bar{\psi}, \delta \phi, w_{y}$, and $w_{z}$. These equations also indicate how the instability mechanism analyzed in the following subsections proceeds intuitively. The external dynamic force, the shear flow $S$, acts as a source term for the velocity difference, $w_{i}$. Inspecting the dynamic Eqs. (58), (60), and (61), we see that relative velocity and concentration $\phi$ are coupled. In the following we will show that the feedback between $w_{i}$ and $\phi$ can start an instability for large enough magnitude of the driving force $S$ leading to spatially and temporally varying nonzero values for $w_{i}$ and $\delta \phi$. Those in turn trigger timedependent behavior of the director angle $\theta$ and the smectic degree of order $\psi$ via the dynamic Eqs. (55) and (56).

\section{B. Linear instability of the flow alignment state with the smectic density as a conserved variable}

It is easily checked that the stationary homogeneous flow alignment state with $w_{i} \equiv 0$ is always a solution. The challenge is to find a regime of the driving force $S$, where this solution is becoming unstable giving way to a generally timedependent, inhomogeneous solution with a nonzero $w_{i}$. It appears to be rather difficult to find such a solution satisfying all five of the nonlinear coupled partial differential equations simultaneously, even the already simplified ones, Eq. (53), (56), (58), and (59).

Therefore, we will consider the special case that the $\hat{\beta}_{i j}$ tensor can be neglected. In that case the variables $\phi$ and $\theta$ are decoupled from the others and we can analyze the linear stability of the three Eqs. (58), (60), and (61). We will investigate for which values of the external driving force, the shear $S$, the solution $w \equiv 0$ becomes unstable. Along the lines of a linear stability analysis as it is done frequently for the onset of a hydrodynamic instability, for example, for the onset of thermal convection from the heat conduction state, we start with the plane wave ansatz,

$$
\left(\phi, w_{y}, w_{z}\right)=\left(\Phi, W_{y}, W_{z}\right) \exp \left(D t+\left[i \omega t+i k_{y} y+i k_{z} z\right]\right),
$$

with free amplitudes $\Phi, W_{y}, W_{z}$. We look for which values of $\omega$ and $S$ we find a solution with $D \equiv 0$, where $\left(\phi, w_{y}, w_{z}\right)$ do not decay to zero anymore. Here we will assume that $k_{y}$ is not 
fixed externally, but must be optimized, while $k_{z}$ has a fixed value to accommodate the conditions at top and bottom plate of the shear setup, e.g., $k_{z}=\pi / L$ with $L$ the thickness of the layer. This procedure closely parallels that of Ref. [50] where the instability of shear flows in nematics has been studied.

Inserting the ansatz Eq. (64) into Eqs. (58) and (60) we obtain for $D=0$ the two coupled equations

$$
\begin{gathered}
i \omega^{3}=\left(\xi^{2} \alpha^{2}+\frac{\alpha}{\kappa_{\phi} \rho^{2}} k^{2}+2 \frac{d_{\perp} \alpha \xi}{\kappa_{\phi}} k^{2}\right) i \omega, \\
2 \omega^{2} \xi \alpha=\frac{\xi \alpha^{2}}{\kappa_{\phi} \rho^{2}} k^{2}-S k_{z} k_{y} \frac{\alpha}{\kappa_{\phi} \rho^{2}}+\frac{d_{\perp}}{\kappa_{\phi}}\left(\alpha^{2} \xi^{2}-\omega^{2}\right) k^{2},
\end{gathered}
$$

with $k^{2}=k_{y}^{2}+k_{z}^{2}$. To simplify the formulas, we have approximated $d_{i j}$ by its isotropic form $d_{\perp} \delta_{i j}$. Equation (65) allows for two solutions, $\omega=0$ and $\omega \neq 0$, which give rise to a stationary and an oscillatory instability, respectively.

In the stationary case, minimizing of $S$ in Eq. (66) with respect to $k_{y}$ leads to the critical value of $k_{y}, k_{y}^{\mathrm{cs}}$,

$$
k_{y}^{\mathrm{cs}}=S k_{z} \frac{1}{2 \xi \alpha}\left(1+\xi d_{\perp} \rho^{2}\right)^{-1} .
$$

Inserting $k_{y}^{\mathrm{cs}}$ into Eq. (66) we obtain the critical shear $S_{\mathrm{cs}}^{2}$

$$
S_{\mathrm{cs}}^{2}=4 \alpha^{2} \xi^{2}\left(1+\xi d_{\perp} \rho^{2}\right)^{2} .
$$

for the onset of a stationary, space-dependent state, with nonzero $w$.

In the oscillatory case, combining Eqs. (65) and (66) and minimizing with respect to $k_{y}$ we obtain for the critical value of $k_{y}, k_{y}^{\mathrm{co}}$,

$$
k_{y}^{\mathrm{co}} \approx-S k_{z} \frac{1}{2 \xi \alpha}\left(1+4 \xi d_{\perp} \rho^{2}\right)^{-1} .
$$

This approximate result is obtained by disregarding fourth order terms in the wave vector. Results for the case when only terms $\sim k_{y}^{4}$ are neglected, corresponding to a long wavelength instability in the plane of the sample, are listed in Appendix B.

Inserting $k_{y}^{\text {co }}$ into Eqs. (65) and (66) we obtain for the critical shear $S_{\text {co }}^{2}$

$$
S_{\mathrm{co}}^{2}=4 \alpha^{2} \xi^{2}\left(1+4 \xi d_{\perp} \rho^{2}\right)^{2}+\frac{8}{k_{z}^{2}} \alpha^{3} \rho^{2} \kappa_{\phi} \xi^{4}\left(1+4 \xi d_{\perp} \rho^{2}\right),
$$

and for the critical frequency at onset

$$
\omega_{c}^{2}=3 \alpha^{2} \xi^{2} \frac{1+\frac{8}{3} \xi d_{\perp} \rho^{2}}{1+4 \xi d_{\perp} \rho^{2}}+k_{z}^{2} \frac{2 \alpha}{\kappa_{\phi} \rho^{2}}\left(1+2 \xi d_{\perp} \rho^{2}\right) .
$$

Comparing Eqs. (67) and (68) to Eqs. (69) and (70) we make the following observations. In general, $S_{\mathrm{co}}^{2}>S_{\mathrm{cs}}^{2}$, meaning that the stationary instability comes first, when increasing the driving force $S$. Second, the critical shear rate for the stationary instability does not depend on $k_{z}$, while for the oscillatory instability it does. Third, the dependence of the critical shear rate of the instability on $k_{z}$ leads to a higher threshold for thicker samples and finally the length scale of the transverse pattern is larger in the oscillatory case, since $\left|k_{y}^{\mathrm{co}}\right|<\left|k_{y}^{\mathrm{cs}}\right|$.
From the results of this simplified, two-dimensional model several observations emerge. First of all the threshold shear is essentially determined by the dissipative effects involved. The first one is the relaxation of the relative velocity $w_{i}$, with the associated dissipative channel $\sim \xi$. Its inverse also sets the characteristic time dependence for the velocity difference $w_{i}$-large relaxation times correspond to velocity differences arising on a long timescale. Second, the diffusion of the concentration $\sim d_{\perp}$ enters the critical quantities and always in the combination $\sim \xi d_{\perp} \rho^{2}$, where $\rho$ is the total density. In addition, the critical quantities are proportional to various powers of the generalized susceptibility $\alpha \equiv \phi(1-\phi) \rho$, connecting the conjugate field of the relative velocity, $m_{i}$, to the relative velocity, $w_{i}$.

Taking into account the relaxation of $\phi$ naturally further increases the threshold value of $S$ as will be shown in Sec. IV C.

It is very likely that for more realistic models, complicated space- and time-dependent instabilities emerge above the linear instability threshold. There, more degrees of freedom become available to trigger such higher instabilities, in particular in three dimensions. We also note that the linear stability analysis presented could be pre-emptied by finite amplitude instabilities. From the experimental results available we know empirically that time-dependent behavior-tumbling, irregular and chaotic oscillations-dominates [24,25,29].

Inserting the space- and time-dependent solutions for $w_{i}$ and $\phi$ into Eqs. (55) and (57) naturally gives rise to a spaceand time-dependence of the director field, $n_{i}$, and the smectic degree of order, $\psi$, as well. This is in accord with experimental observations of a time-dependent director field at the onset of tumbling. As usual, the linear instability analysis relates their amplitudes $\Theta$ and $\Psi$ to the other amplitudes $\Phi, W_{y}, W_{z}$, where, however, one amplitude remains undetermined and has to be fixed by a nonlinear treatment.

To make a quantitative comparison with experiments it will be important to pin down more precisely as many of the static and transport parameters involved as possible.

\section{Linear instability of the flow alignment state with the smectic density as a relaxing variable}

In the last section we have assumed the different mass densities of the nematic $\rho_{n}$ and smectic parts $\rho_{s}$ change by mutual diffusion, i.e., both are conserved quantities. This means that $\rho_{s}$ and $\rho_{n}$ are redistributed in space and time in a diffusive manner. However, as relaxing variables $\rho_{s}$ and $\rho_{n}$ can appear and disappear without having to diffuse, and thus no transport is involved. Which one of the two cases applies to experiment is not known to date. It might actually depend on the class of materials involved. For example, for a liquid crystalline polymer diffusion over finite distances is most likely more difficult than for small molecules. Therefore, we discuss both possibilities in the present paper and consider in this section the possibility that the densities are slowly relaxing variables.

Assuming the densities to relax we have

$$
\dot{\rho}_{s}=\cdots-\frac{1}{\tau_{s}} \mu_{s} \quad \text { and } \quad \dot{\rho}_{n}=\cdots-\frac{1}{\tau_{n}} \mu_{n},
$$

according to the appropriate conjugates $\mu_{s}$ and $\mu_{n}$. The dots in Eq. (72) denote all the conserved contributions. Since the 
total density $\rho$, being a conserved variable, must not relax, we have to require the condition $\mu_{s} / \tau_{s}+\mu_{n} / \tau_{n}=0$. Expressing the conjugates $\mu_{s}$ and $\mu_{n}$ by the conjugates $\Pi$ and $\mu$ of the concentration $\phi$ and the total density $\rho$, respectively [1],

$$
\mu_{s}=\mu+(1-\phi) \Pi / \rho \text { and } \mu_{n}=\mu-\phi \Pi / \rho,
$$

we can write the parts of the entropy production associated with the relaxations of Eq. (72) as

$$
\begin{aligned}
2 R & =\frac{1}{\tau_{s}} \mu_{s}^{2}+\frac{1}{\tau_{n}} \mu_{n}^{2} \\
& =\frac{1}{\tau_{s}}(\mu+[1-\phi] \Pi / \rho)^{2}+\frac{1}{\tau_{n}}(\mu-\phi \Pi / \rho)^{2} .
\end{aligned}
$$

Since $\dot{\rho}$ cannot show any relaxation, there is $\partial R / \partial \mu=0$ leading to $\left(\tau_{n}+\tau_{s}\right) \mu=\left(\tau_{s} \phi-\tau_{n}[1-\phi]\right) \Pi / \rho$. As a result

$$
2 R=\frac{1}{\tau_{s}+\tau_{n}}(\Pi / \rho)^{2} \equiv \frac{\phi(1-\phi)}{\tau_{\phi}} \Pi^{2}=\frac{\alpha}{\rho \tau_{\phi}} \Pi^{2},
$$

with $\tau_{\phi}=\phi(1-\phi) \rho^{2}\left(\tau_{n}+\tau_{s}\right)$, where we have made apparent the $\phi$ factors to make sure that there is no relaxation for $\phi=0$ and $\phi=1$. Finally Eq. (58) takes the form

$$
\dot{\phi}+\frac{\alpha}{\rho} \nabla_{i} w_{i}-\frac{1}{\kappa_{\phi}} d_{i j} \nabla_{i} \nabla_{j} \phi+\frac{\alpha}{\rho \tau_{\phi} \kappa_{\phi}} \delta \phi=0 .
$$

We now investigate the stationary and oscillatory instability taking into account the relaxation of $\phi$, but neglecting the diffusion $\nabla_{i} \nabla_{j} \phi$. Using the same procedure as in Sec. IV B, the two equations

$$
\begin{gathered}
i \omega^{3}=\left(\xi^{2} \alpha^{2}+\frac{\alpha}{\kappa_{\phi} \rho^{2}}\left(k_{y}^{2}+k_{z}^{2}\right)+2 \xi \frac{\alpha^{2}}{\rho \tau_{\phi} \kappa_{\phi}}\right) i \omega, \\
2 \omega^{2} \xi=\xi \frac{\alpha}{\kappa_{\phi} \rho^{2}}\left(k_{y}^{2}+k_{y}^{2}\right)-S k_{z} k_{y} \frac{1}{\kappa_{\phi} \rho^{2}} \\
+\frac{1}{\rho \tau_{\phi} \kappa_{\phi}}\left(-\omega^{2}+\alpha^{2} \xi^{2}\right)
\end{gathered}
$$

allow for a stationary instability at

$$
S_{\mathrm{cs}}^{2}=4 \alpha^{2} \xi^{2}+\frac{4}{k_{z}^{2}} \frac{\alpha^{3} \xi^{3} \rho^{2}}{\rho \tau_{\phi}}
$$

with a critical wave vector independent of $\tau_{\phi}$,

$$
k_{y}^{\mathrm{cs}}=S k_{z} \frac{1}{2 \xi \alpha} .
$$

In addition, for the oscillatory instability we find the critical wave vector

$$
k_{y}^{c o}=-\frac{k_{z} S}{2 \alpha \xi}(1+M)^{-1},
$$

with the abbreviation $M=1 /\left(\rho \kappa_{\phi} \tau_{\phi} \xi\right)$, the critical shear flow

$$
S_{c o}^{2}=4 \alpha^{2} \xi^{2}(1+M)^{2}+\frac{8}{k_{z}^{2}} \rho^{2} \kappa_{\phi} \alpha^{3} \xi^{4}(1+M)^{3},
$$

and the critical frequency at onset

$$
\omega_{c}^{2}=\alpha^{2} \xi^{2}(3+4 M)+\frac{2 \alpha}{\kappa_{\phi} \rho^{2}} k_{z}^{2} .
$$

We remark that all three critical quantities [Eqs. (81)-(83)] for the oscillatory instability have a characteristic dependence on $M=1 /\left(\rho \kappa_{\phi} \tau_{\phi} \xi\right)$, which contains the generalized susceptibility $\kappa_{\phi}$ connecting the conjugate force of the phase, $\Pi$, with the phase variations, $\delta \phi$, as well as the $\xi$ and $\tau_{\phi}$, describing the relaxation of $\delta \phi$ and of the relative velocity, $w_{i}$, respectively. In addition we notice that the stationary wave vector $k_{y}^{\mathrm{cs}}$ [Eq. (80)] does not depend on $\tau_{\phi}$ in contrast to $k_{y}^{\text {co }}$ [Eq. (81)].

Comparing with Sec. IV B the following remarks are in order. (i) The role of the diffusion coefficient $d_{\perp}$ is taken over by the inverse relaxation time $1 / \tau_{\phi}$. (ii) Both quantities enter the stationary and oscillatory threshold values for the critical shear rate: Larger values of $\tau_{\phi}$ and smaller values of $d_{\perp}$ lead to a reduction of the threshold values. (iii) The dependence of the oscillatory threshold value on $1 / k_{z}^{2}$, and of the critical frequency on $k_{z}^{2}$, is similar for the diffusing and relaxing case. A difference arises for the stationary instability threshold, which does not depend on $k_{z}$ in the diffusing case, in contrast to Eq. (79). The wave vector dependence governs the dependence on the sample thickness $D$, since $k_{z} \sim k_{y} \sim 1 / D$ for all cases.

\section{SUMMARY AND PERSPECTIVE}

In this work we have analyzed the macroscopic dynamics of nematic liquid crystals in the presence of smectic clusters under an applied shear flow. We have shown that the macroscopic dynamics for this system with only one velocity field is insufficient to describe time-dependent director dynamics due to the presence of smectic clusters, since there is no coupling between the director orientation and the flow induced smectic order. In contrast, we have demonstrated that a twofluid model leads to a coupling between shear flow induced smectic order and the macroscopic director orientation. This interaction is mediated by the velocity difference between the two subsystems. In particular, we have shown the existence of transitions from the homogeneous flow alignment state to space-dependent states, being either static or time-dependent, when the external shear is increased. It also emerges that for this problem one needs for a consistent description at least two macroscopic variables, which relax on a sufficiently long, but finite timescale, namely, the velocity difference and the degree of smectic order. Both variables are neither related to conservation laws nor to spontaneously broken continuous symmetries. Both instabilities are found for a diffusive as well as for a relaxing smectic mass density.

We notice some relationship of the present problem to the macroscopic dynamics above the $\lambda$-transition in superfluid ${ }^{4} \mathrm{He}$, where one has a relaxing superfluid density, $\rho_{s},[7,51]$, which plays a similar role as the relaxing degree of smectic order, $\psi$, used here. In particular, a contribution similar to $\dot{\psi} \sim \beta_{i j} A_{i j}$ in Eq. (56) arises in Ref. [7] in the $\dot{\rho}_{s}$ equation. As a difference, our system is uniaxial, while the superfluid system is isotropic. Another importance difference is manifest in the superfluid velocity, which is a hydrodynamic variable in ${ }^{4} \mathrm{He}$ due to the spontaneously broken gauge symmetry.

We also point out that our analysis can be used to describe the breakdown of flow alignment of the in-plane director inside fluid smectic layers, when bond-orientational order enters the picture. The geometry of choice to demonstrate this 
behavior are freely suspended smectic C films with bondorientational clusters. This type of clusters can definitely expected to occur above smectic F, I, and L phases [42-44].

Throughout the present paper we have studied the influence of smectic clusters and clusters of bond-orientational order thus focusing on the influence of layering in a broad sense. We would like to point out, however, that a very similar type of behavior should arise in systems, which have a tendency toward column formation. We are thinking in particular of columnar fluctuations above a nematic to columnar transition [41]. We also note that the occurrence of the influence of smectic or columnar fluctuations could arise as well when the temperature is increased, namely, for reentrant nematics in smectic systems [52,53] or for inverted and/or reentrant columnar phases [38-40]. In both cases we expect timedependent director motion for growing temperatures.

\section{ACKNOWLEDGMENTS}

Partial support of this work by the Deutsche Forschungsgemeinschaft through the Schwerpunktprogramm SPP 1681 "Feldgesteuerte Partikel-Matrix-Wechselwirkungen: Erzeugung, skalenübergreifende Modellierung und Anwendung magnetischer Hybridmaterialien" is gratefully acknowledged.

\section{APPENDIX A: SIMPLE MODEL FOR THE SIGN CHANGE OF THE DIELECTRIC ANISOTROPY}

The most important macroscopic effect-aside from the breakdown of flow alignment-signaling the presence of smectic clusters is the sign change in the anisotropy of the electric conductivity, $\sigma_{a}=\sigma_{\|}-\sigma_{\perp}$ [31-34]. Here and throughout this Appendix $\|$ and $\perp$ denote the directions parallel and perpendicular to the director. In a nematic phase without smectic clusters, $\sigma_{a}$ is positive meaning physically that the transport of charges parallel to the director $n_{i}$ is easier than along the perpendicular direction and, therefore $\sigma_{\|}>\sigma_{\perp}$. As the volume fraction of smectic clusters grows when a sample is cooled down, a sign change in $\sigma_{a}$ arises. Intuitively, this opposite behavior compared to the case of a usual nematic occurs, because the charge transport across smectic layers is much harder than within smectic layers.

In this Appendix we analyze how this qualitative change in behavior can be accounted for in a simple two-fluid model, where the two velocities are the average velocities of the charge carriers in a smectic and in a nematic environment, respectively.

First we elucidate some macroscopic aspects. Denoting by $\sigma_{\|}^{n}$ and $\sigma_{\perp}^{n}$ the electric conductivities in a pure nematic and by $\sigma_{\|}^{s}$ and $\sigma_{\perp}^{s}$ in a pure smectic $A$ phase, we will assume that we can make an effective medium approximation to obtain the conductivities of a nematic phase with smectic clusters. This means that we make the ansatz

$$
\begin{gathered}
\sigma_{\|}=\phi \sigma_{\|}^{n}+(1-\phi) \sigma_{\|}^{s}, \\
\sigma_{\perp}=\phi \sigma_{\perp}^{n}+(1-\phi) \sigma_{\perp}^{s},
\end{gathered}
$$

From Eqs. (A1) it is straightforward to evaluate the critical concentration, $\phi_{c}$, for which $\sigma_{\|}-\sigma_{\perp}=\sigma_{a} \equiv 0$ with the result

$$
\phi_{c}=\frac{\sigma_{a}^{s}}{\sigma_{a}^{s}-\sigma_{a}^{n}} .
$$

Note that $0<\phi_{c}<1$, since $\sigma_{a}^{s}<0$.

Now we will add a simple mesoscopic model for the conductivity of an isotropic, charged liquid with charge $q$ and mobility $\mu$, where the conductivity $\sigma$ is given by

$$
\sigma=\mu q^{2}
$$

with the mobility, $\mu \sim v l$, expressed by $v$ and $l$ [54], the average velocity and the mean free path of the charge carriers, respectively. The proportionality factor is $\gamma=1 /\left(3 k_{B} T\right)$. In the uniaxially anisotropic case we can write accordingly,

$$
\frac{\sigma_{\|}}{\sigma_{\perp}}=\frac{v_{\|} l_{\|}}{v_{\perp} l_{\perp}}
$$

Since the electric conductivity tensor $\sigma_{i j}$ is associated with a linear material law $\left(j_{i}=\sigma_{i j} E_{j}\right)$ within linear response theory, it appears natural to make for a nematic liquid crystal with smectic clusters the linear superposition ansatz

$$
\begin{aligned}
\sigma_{\|} & =\gamma\left([1-\phi] v_{\|}^{s} l_{\|}^{s}+\phi v_{\|}^{n} l_{\|}^{n}\right), \\
\sigma_{\perp} & =\gamma\left([1-\phi] v_{\perp}^{s} l_{\perp}^{s}+\phi v_{\perp}^{n} l_{\perp}^{n}\right) .
\end{aligned}
$$

For $\sigma_{a}^{n}$ and $\sigma_{a}^{s}$ we then have

$$
\begin{aligned}
\sigma_{a}^{n} & =\gamma \phi\left(v_{\|}^{n} l_{\|}^{n}-v_{\perp}^{n} l_{\perp}^{n}\right), \\
\sigma_{a}^{s} & =\gamma(1-\phi)\left(v_{\|}^{s} l_{\|}^{s}-v_{\perp}^{s} l_{\perp}^{s}\right) .
\end{aligned}
$$

Combining the macroscopic and mesoscopic aspects of picture suggested here, Eqs. (A1) and (A5), we obtain a direct relation between the critical value of the concentration, $\phi_{c}$, for which the conductivity anisotropy changes sign, in terms of four components of the mean velocities of the charge carriers and their appropriate mean free paths,

$$
\phi_{c}=\frac{v_{\perp}^{s} l_{\perp}^{s}-v_{\|}^{s} l_{\|}^{s}}{v_{\|}^{n} l_{\|}^{n}-v_{\perp}^{n} l_{\perp}^{n}+v_{\perp}^{s} l_{\perp}^{s}-v_{\|}^{s} l_{\|}^{s}} .
$$

It will be most interesting to check experimentally to what extent Eq. (A7) can be verified for a real system.

\section{APPENDIX B: THE CASE OF A LONG WAVELENGTH INSTABILITY IN THE PLANE OF THE SAMPLE WHEN THE SMECTIC MASS DENSITY $\rho_{s}$ IS CONSERVED}

Here we assume $k_{y}^{2} \ll k_{z}^{2}$, which allows to neglect the contribution $\sim k_{y}^{4}$. In that case we get for the oscillating instability

$$
\begin{gathered}
k_{y}^{c o a} \approx-S k_{z} \frac{1}{2 \xi \alpha N}, \\
S_{c o a}^{2}=4 \alpha^{2} \xi^{2} N^{2}+\frac{8}{k_{z}^{2}} \alpha^{3} \rho^{2} \kappa_{\phi} \xi^{4} N \\
\omega_{c a}^{2}=\alpha^{2} \xi^{2}\left(1+2 \frac{1+2 \xi d_{\perp} \rho^{2}}{N}\right)+k_{z}^{2} \frac{2 \alpha}{\kappa_{\phi} \rho^{2}}\left(1+2 \xi d_{\perp} \rho^{2}\right),
\end{gathered}
$$

with the abbreviation

$$
N=1+4 \xi d_{\perp} \rho^{2}+k_{z}^{2} \frac{2 d_{\perp}}{\kappa_{\phi} \alpha \xi}\left(1+2 \xi d_{\perp} \rho^{2}\right) .
$$

where $\phi$ is the concentration of nematics. 
[1] H. Pleiner and J. L. Harden, Natural Sciences Special Issue: Nonlinear Problems of Continuum Mechanics (Notices of Universities, South of Russia, 2003), pp. 46-61.

[2] H. Pleiner and J. L. Harden, in Slow Dynamics in Complex Systems: 3rd International Symposium on Slow Dynamics in Complex Systems, edited by M. Tokuyama and I. Oppenheim, AIP Conf. Proc. No. 708 (AIP, New York, 2004), p. 46.

[3] H. Pleiner, A. M. Menzel, and H. R. Brand (unpublished).

[4] H. Pleiner, D. Svenšek, and H. R. Brand, Eur. Phys. J. E 36, 135 (2013).

[5] H. Pleiner, D. Svenšek, and H. R. Brand, Rheol. Acta 55, 857 (2016).

[6] H. Pleiner, D. Svenšek, T. Potisk, and H. R. Brand, Phys. Rev. E 101, 032601 (2020).

[7] I. M. Khalatnikov, Introduction to the Theory of Superfluidity (Benjamin, Reading, MA, 1965).

[8] P. C. Hohenberg and P. C. Martin, Ann. Phys. (NY) 34, 291 (1965).

[9] R. Graham, Phys. Rev. Lett. 33, 1431 (1974).

[10] R. Graham and H. Pleiner, Phys. Rev. Lett. 34, 792 (1975).

[11] M. Liu, Phys. Rev. B 13, 4174 (1976).

[12] R. Graham and H. Pleiner, J. Phys. C 9, 279 (1976).

[13] H. R. Brand, M. Dörfle, and R. Graham, Ann. Phys. 119, 434 (1979).

[14] D. Forster, Hydrodynamic Fluctuations, Broken Symmetry, and Correlation Functions (Benjamin, Reading, MA, 1975).

[15] P. G. De Gennes, The Physics of Liquid Crystals (Clarendon Press, Oxford, 1975).

[16] D. Forster, Phys. Rev. Lett. 32, 1161 (1974).

[17] D. Forster, Ann. Phys. (NY) 84, 505 (1974).

[18] P. C. Martin, O. Parodi, and P. S. Pershan, Phys. Rev. A 6, 2401 (1972).

[19] H. Brand and H. Pleiner, J. Phys. (Paris) 43, 853 (1982).

[20] H. Brand and H. Pleiner, J. Phys. (Paris) 44, 754 (1983).

[21] Ch. Gähwiller, Phys. Rev. Lett. 28, 1554 (1972).

[22] Ch. Gähwiller, Mol. Cryst. Liq. Cryst. 20, 301 (1973).

[23] P. Pieranski and E. Guyon, Phys. Rev. Lett. 32, 924 (1974).

[24] P. E. Cladis and S. Torza, Phys. Rev. Lett. 35, 1283 (1975).

[25] P. E. Cladis and S. Torza, in Colloid and Interface Science, edited by M. Kelker, Vol. 4 (Academic Press, New York, 1976), p. 487.

[26] M. G. Kim, S. Park, M. Cooper, and S. V. Letcher, Mol. Cryst. Liq. Cryst. 36, 143 (1976).

[27] W. W. Beens and W. H. de Jeu, J. Phys. (Paris) Lett. 44, 805 (1983).

[28] D. J. Ternet, R. G. Larson, and L. G. Leal, Rheol. Acta 38, 183 (1999).
[29] H. Siebert, I. Quijada-Garrido, J. Vermant, L. Noirez, W. R. Burghardt, and C. Schmidt, Macromol. Chem Phys. 208, 2161 (2007).

[30] P. E. Cladis and H. R. Brand, Physica A 326, 322 (2003).

[31] F. Rondelez, Solid State Comm. 11, 1675 (1972).

[32] A. Mircea-Roussel and F. Rondelez, J. Chem. Phys. 63, 2311 (1975).

[33] J. Jadzyn and P. Kedziora, Mol. Cryst. Liq. Cryst. 145, 17 (1987).

[34] H. R. Brand, C. Fradin, P. L. Finn, W. Pesch, and P. E. Cladis, Phys. Lett. A 235, 508 (1997).

[35] K. Skarp and T. Carlsson, Mol. Cryst. Liq. Cryst. 49, 75 (1978).

[36] T. Carlsson and K. Skarp, Mol. Cryst. Liq. Cryst. 78, 157 (1981).

[37] T. Potisk, H. Pleiner, D. Svenšek, and H. R. Brand, Phys. Rev. E 97, 042705 (2018).

[38] C. Destrade, J. Malthète, N. H. Tinh, and H. Gasparoux, Phys. Lett. A 78, 82 (1980).

[39] N. H. Tinh, J. Malthète, and C. Destrade, J. Phys. (Paris) Lett. 42, 417 (1981).

[40] C. Destrade, H. Gasparoux, H. Babeau, N. H. Tinh, and J. Malthète, Mol. Cryst. Liq. Cryst. 67, 37 (1981).

[41] H. R. Brand, Phys. Rev. A 33, 643 (1986).

[42] P. A. C. Gane, A. J. Leadbetter, J. J. Benattar, F. Moussa, and M. Lambert, Phys. Rev. A 24, 2694 (1981).

[43] H. Pleiner and H. R. Brand, Phys. Rev. A 29, 911 (1984).

[44] G. S. Smith, E. B. Sirota, C. R. Safinya, R. J. Plano, and N. A. Clark, J. Chem. Phys. 92, 4519 (1990).

[45] P. E. Cladis, Y. Couder, and H. R. Brand, Phys. Rev. Lett. 55, 2945 (1985).

[46] H. B. Callen, Thermodynamics and an Introduction to Thermostatics (John Wiley \& Sons, New York, 1985).

[47] H. Pleiner and H. R. Brand, in Pattern Formation in Liquid Crystals, edited by A. Buka and L. Kramer (Springer, New York, 1996).

[48] M. Liu, Phys. Rev. A 19, 2090 (1979).

[49] D. Forster, T. C. Lubensky, P. C. Martin, J. Swift, and P. S. Pershan, Phys. Rev. Lett. 26, 1016 (1971).

[50] P. Pieranski and E. Guyon, Phys. Rev. A 9, 404 (1974).

[51] L. P. Pitaevskii, Exptl. Theoret. Phys. (USSR) 35, 408 (1958) [Soviet Physics JETP 8, 282 (1959)].

[52] P. E. Cladis, Phys. Rev. Lett. 35, 48 (1975).

[53] P. E. Cladis, Mol. Cryst. Liq. Cryst. 67, 177 (1981).

[54] R. P. Feynman, R. B. Leighton, and M. Sands, The Feynman Lectures on Physics, Vol. I, Chapt. 43, Eqs. (43.27) and (43.31) (Pearson Addison-Wesley, Reading, MA, 1963). 